\title{
Referral Rates and Costs of Our Modified Two-Step Newborn Hearing Screening Program at a Japanese Perinatal Center
}

\author{
Mieko Sato ${ }^{a}$, Shunji Suzuki ${ }^{\mathrm{a}, \mathrm{b}}$
}

\begin{abstract}
Background: We examined the referral rates and costs of our modified two-step newborn hearing screening program for "low-risk" infants at our hospital from 2004 to 2013.

Methods: In our hospital, low-risk newborns were screened with an otoacoustic emissions (OAE) sensor on days 2 - 4 postpartum. Infants who failed the OAE were examined with OAE again 1 - 5 days after the first screening. Infants who failed the second OAE were screened with the third OAE at 1 month after delivery. Infants who failed the third OAE were examined with auditory brainstem response at $1-2$ weeks after the third OAE.
\end{abstract}

Results: Twenty-eight of 19,199 cases $(0.15 \%)$ had unilateral or bilateral REFER, and 19 cases $(0.10 \%)$ were diagnosed as hearing loss (three cases were missing). Thus, the false positive rate in our programs was $28 \%$. During the study period, the total cost for each program was calculated to be JP¥ 526 .

Conclusions: Our modified two-step newborn hearing screening program for "low-risk" infants seemed to be beneficial concerning their referral rates and costs.

Keywords: Two-step newborn hearing screening; Otoacoustic emissions; Automated auditory brainstem response; Referral rate; Japan

\section{Introduction}

Undetected congenital permanent hearing loss impairs de-

Manuscript accepted for publication June 26, 2014

${ }^{\text {a } D e p a r t m e n t ~ o f ~ O b s t e t r i c s ~ a n d ~ G y n e c o l o g y, ~ J a p a n e s e ~ R e d ~ C r o s s ~}$ Katsushika Maternity Hospital, Tokyo, Japan

${ }^{\mathrm{b}}$ Corresponding Author: Shunji Suzuki, Department of Obstetrics and Gynecology, Japanese Red Cross Katsushika Maternity Hospital,

5-11-12 Tateishi, Katsushika-ku, Tokyo 124-0012, Japan.

Email: czg83542@mopera.ne.jp

doi: http://dx.doi.org/10.14740/ijcp155w velopment in infants by diminishing their ability to acquire language and cognitive skills at an appropriate age [1]. Current estimates have been reported to be that one to six per 1,000 infants are born with significant permanent hearing loss $[1,2]$. Universal newborn hearing screening (UNHS) is an effective way of identifying hearing loss in newborn, and in conjunction with initiation of appropriate intervention within 6 months of diagnosis of hearing loss in infants, it is shown to result in the development of significantly better language abilities, compared with infants identified with hearing loss later on in life [1-5]. Therefore, UNHS programs are now accepted worldwide to be beneficial to prevent the delay in speech-language development. For example, 90\% of all newborns in the United States have been reported to be screened for hearing loss before they leave the hospital [3].

Based on the principles and guidelines for early hearing detection and intervention programs in the United States $[3,4]$, for example, newborns in alternative birthing facilities, including home births, have access to and are referred for screening before 1 month of age as UNHS programs. All infants who do not pass the birth admission screen and any subsequent rescreening begin appropriate audiologic and medical evaluations to confirm the presence of hearing loss before 3 months of age. All infants with confirmed permanent hearing loss receive services before 6 months of age.

There have been three commonly used protocols available for UNHS programs: 1) otoacoustic emissions (OAE); 2) automated auditory brainstem response (AABR); and (3) a "two-step" program in which infants are first screened with OAE, followed by an AABR screen for those infants who do not pass the OAE screen [6]. Table 1 shows the differences among OAE, AABR and auditory brainstem response (ABR) as a newborn hearing screening test (JP $¥ 100=$ US\$ 1). The referral rate of screening with AABR is lower than that of OAE; however, the screening with AABR is costly [7]. Although the screening with ABR has low referral rates and low costs, it takes about $30 \mathrm{~min}$ for one screening. These differences in referral rates and/or costs of UNHS are very important because there are no or few public contributions to the UNHS programs in most areas of Japan [8]. Although Japan might be a wealthy country, Katsushika area in Tokyo is not wealthy. Based on these backgrounds, we examined 
Table 1. Differences Among OAE, AABR and ABR as a Newborn Hearing Screening Test

\begin{tabular}{llll}
\hline & OAE & AABR & ABR \\
\hline Extraction sensitivity of hearing loss & About $100 \%$ & $\begin{array}{l}95-98 \% \text { (near } 100 \% \text { in low } \\
\text { risk infants) }\end{array}$ & About $100 \%$ \\
Time required for inspection & $10-30 \mathrm{~s}$ & $30 \mathrm{~s}-15$ min & 30 min \\
Price of equipment & JP¥ 1,500,000/6 years & JP¥ 3,300,000/6 years & JP¥ 2,635,000/6 years \\
Price of consumables & JP¥ 100 & JP¥ 2,500 & JP¥ 10 \\
\hline
\end{tabular}

JP¥ $¥ 100=$ US\$ 1.

the referral rates and costs of our modified two-step newborn hearing screening program for "low-risk" infants at our institute, one of main perinatal centers in Katsushika area, Tokyo, Japan (1,900 - 2,000 deliveries per year). This incorporated two additional/optional OAE tests. Infants not passing OAE tests were referred to the next screening test with ABR.

\section{Methods}

We examined the "low-risk" infants who were born in Japanese Red Cross Katsushika Maternity Hospital from 2004 to 2013. At our hospital, all mothers were informed of the role and significance of newborn hearing screening before birth. All examinations were performed by one experienced examiner (M.S.). Every family incurred JP¥ 3,000 as the cost of newborn hearing screening tests, irrespective of presence or absence of risk factors of hearing loss. After obtaining the family's consents, infants who had risk factors of hearing loss based on the Position Statements from the Joint Committee on Infant Hearing [3, 4, 9] were screened with an Neuropack $\mu^{\circledR}$, an ABR sensor before they left the hospital as "high-risk" newborns. Infants who did not pass the ABR were referred to the specialists of hearing loss.

On the other hand, "low-risk" newborns were screened with an Echo-Screen ${ }^{\circledR}$, a transiently evoked OAE (TEOAE) sensor after obtaining the family's consents. The first screening test with the TEOAE was performed on days 2 - 4 postpartum. No additional testing was done with infants who passed the TEOAE, but infants who failed the TEOAE were examined with TEOAE again 1 - 5 days after the first screening (before they left the hospital). Infants who failed the second TEOAE were screened with the third TEOAE at 1 month after delivery. Infants who failed the third OAE were examined with ABR at $1-2$ weeks after the third OAE. The infants who did not pass the ABR were referred to the specialists of hearing loss before 3 months of age.

In this study, we calculated the referral rates, false positive rates and costs of our modified two-step newborn hearing screening program.

\section{Results}

Table 2 shows the referral rates of our modified two-step protocol with repeat OAE and ABR. Thus, the final referral

Table 2. Referral Rates of Our Modified Two-Step Protocol With Repeat OAE and ABR

\section{REFER}

$\begin{array}{lc}\text { First OAE } & 750(3.9 \%) \\ \text { Second OAE } & 128(0.67 \%) \\ \text { Third OAE } & 81(0.42 \%) \\ \text { ABR } & 28(0.15 \%)\end{array}$




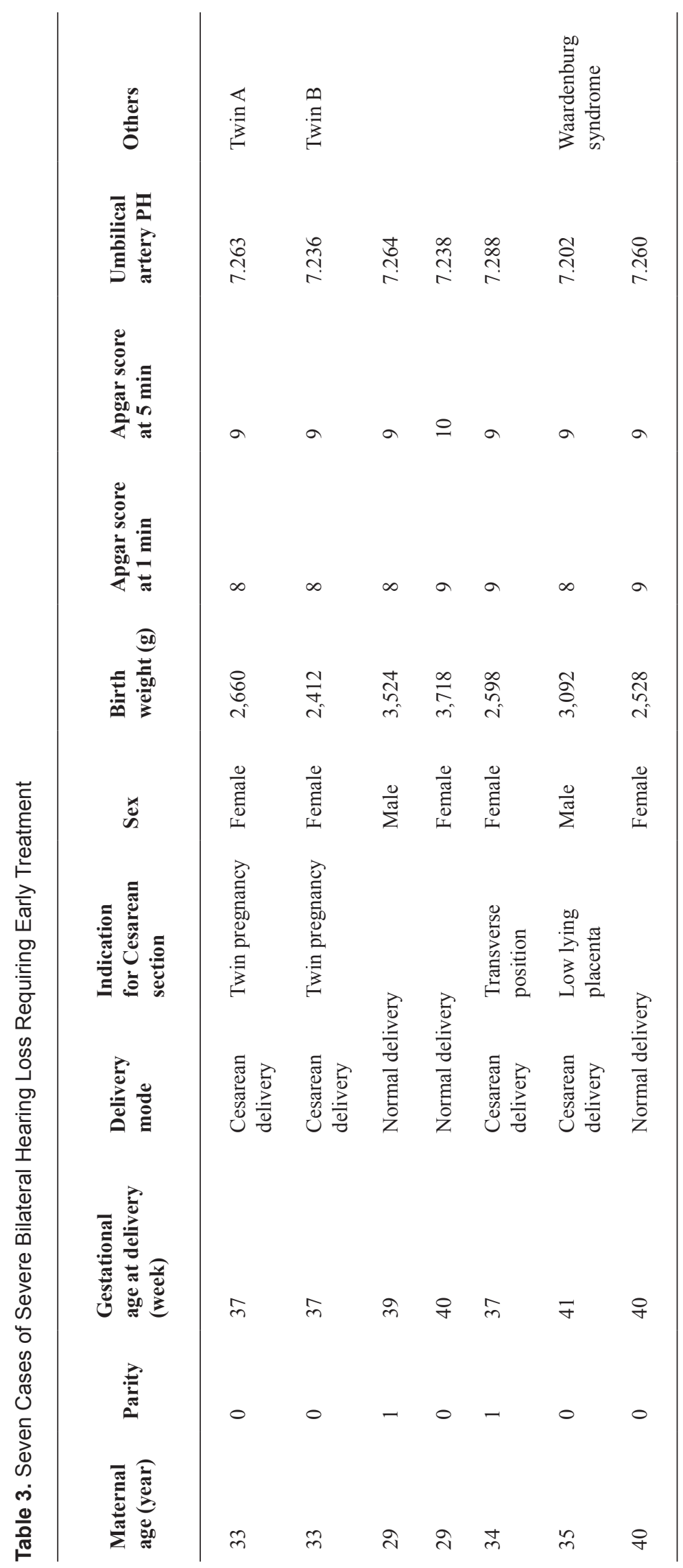


rate was $0.15 \%(28 / 19,199$, eight males and 20 females). Of the 28 cases, 19 cases $(0.10 \%$ of the total examined, five males and 14 females) had unilateral REFER and nine cases $(0.047 \%$ of the total examined, three males and six females) had bilateral REFER. Of these, three cases were missing, seven cases shown in Table $3(0.036 \%$ of the total examined, two males and five females) were diagnosed as severe bilateral hearing loss requiring early treatment and one case $(0.0052 \%$ of the total examined, one female) was diagnosed as moderate bilateral hearing loss requiring follow-up. While, ten cases $(0.052 \%$ of the total examined, three males and seven females) were diagnosed as unilateral hearing loss requiring follow-up. Thus, the false positive rate in our programs was $28 \%$.

During the study period, the total costs for consumables for our two-step program with ABR following repeat $\mathrm{OAE}$ were JP $¥ 3,213,150$ (OAE, JP¥ $1,500,000 \times 10 / 6$ plus 160 $\times 19,199+750+128$ times; ABR, JP¥ 2,635,000 $\times 10 / 6$ plus $10 \times 83$ times). Thus, the total cost for our program was JP¥ $10,104,816$. It is estimated that it took JP $¥ 53,497,500$ if the program was running with only AABR (JP $¥ 3,300,000$ $\times 10 / 6$ plus $2,500 \times 19,199$ times). Therefore, the costs for each newborn hearing screening by our program vs. AABR program were JP¥ 526 vs. 2,786.

\section{Discussion}

UNHS is considered beneficial and is accepted worldwide. However, we also know that some problems remain, and the administrative systematization has yet to be established in Japan $[7,8,10]$. Early public support is also required for infants with either severe to profound or moderate hearing loss. Although the screening should be performed within the first 3 months of infant's life, it is considered that the UNHS protocol considering the timing of screening needs further discussion from the viewpoint of test conditions and puerperal parental psychological problems $[7,8,10]$.

To date, some examinations have been performed to compare the initial referral rate, the accurate identification rate of congenital hearing loss, and the cost between onestep with OAE, two-step with OAE and AABR and one-step with $\mathrm{AABR}$ in newborn hearing screening program $[11,12]$. In an earlier study by Lin et al [11], the total costs, including expenditures and intangible costs, have been observed to be much lower in the protocol with AABR due to reduction in false positives. However, we believe that our current program with $\mathrm{ABR}$ following repeat $\mathrm{OAE}$ could reduce the costs in newborn hearing screening program further due to reduction in false positive rate as same as the protocol with AABR for low-risk newborns previously reported $(0.49 \%)$ [7]. In addition, it is supposed that the current program is a very effective method in the poverty areas in Japan without public expenditures for newborn hearing screening, al- though both the description of the protocol to the mother and the execution of the programs need hard working.

At last, we have to concern the possibility of few cases with AABR fail/OAE pass such as auditory neuropathy [7, 13]. Although the cases have been observed to be associated with severe asphyxia, hyperbilirubinemia and cytomegalovirus infection, the pathophysiology of the cases is not well established [7]. Therefore, the follow-up of hearing of all newborns should be needed in the routine health care.

In conclusions, our modified two-step newborn hearing screening program for "low-risk" infants at our institute seemed to be beneficial concerning their referral rates and costs.

\section{References}

1. Erenberg A, Lemons J, Sia C, Trunkel D, Ziring P. Newborn and infant hearing loss: detection and intervention. American Academy of Pediatrics. Task Force on Newborn and Infant Hearing, 1998-1999. Pediatrics. 1999;103(2):527-530.

2. Vohr BR, Oh W, Stewart EJ, Bentkover JD, Gabbard S, Lemons J, Papile LA, et al. Comparison of costs and referral rates of 3 universal newborn hearing screening protocols. J Pediatr. 2001;139(2):238-244.

3. Joint Committee on Infant Hearing of the American Academy of Pediatrics, Muse C, Harrison J, YoshinagaItano C, Grimes A, Brookhouser PE, Epstein S, et al. Supplement to the JCIH 2007 position statement: principles and guidelines for early intervention after confirmation that a child is deaf or hard of hearing. Pediatrics. 2013;131(4):e1324-1349.

4. Joint Committee on Infant Hearing, American Academy of Audiology, American Academy of Pediatrics, American Speech-Language-Hearing Association, Directors of Speech and Hearing Programs in State Health and Welfare Agencies. Year 2000 position statement: principles and guidelines for early hearing detection and intervention programs. Pediatrics. 2000;106(4):798-817.

5. Qi B, Cheng X, En H, Liu B, Peng S, Zhen Y, Cai Z, et al. Assessment of the feasibility and coverage of a modified universal hearing screening protocol for use with newborn babies of migrant workers in Beijing. BMC Pediatr. 2013;13:116.

6. Johnson JL, White KR, Widen JE, Gravel JS, James M, Kennalley T, Maxon AB, et al. A multicenter evaluation of how many infants with permanent hearing loss pass a two-stage otoacoustic emissions/automated auditory brainstem response newborn hearing screening protocol. Pediatrics. 2005;116(3):663-672.

7. Mishina J. Newborn hearing screening program: a review (in Japanese). J Jpn Pediatr Soc. 2004;108:1449- 
1453.

8. Japan Association of Obstetricians and Gynecologists. The manual for neonatal hearing screening. 2007. http:// www.jaog.or.jp/sep2012/JAPANESE/jigyo/JYOSEI/ shinseiji_html/shi-9.html.

9. Joint Committee on Infant Hearing. Position Statements from the Joint Committee on Infant Hearing. http:// www.jcih.org/posstatemts.htm.

10. Wada T, Kubo T, Aiba T, Yamane H. Further examination of infants referred from newborn hearing screening. Acta Otolaryngol Suppl. 2004;(554):17-25.

11. Lin HC, Shu MT, Lee KS, Lin HY, Lin G. reducing false positives in newborn hearing screening program: how and why. Otol Neurotol. 2007;28(6):788-792.

12. Benito-Orejas JI, Ramirez B, Morais D, Almaraz A, Fernandez-Calvo JL. Comparison of two-step transient evoked otoacoustic emissions (TEOAE) and automated auditory brainstem response (AABR) for universal newborn hearing screening programs. Int J Pediatr Otorhinolaryngol. 2008;72(8):1193-1201.

13. Berg AL, Prieve BA, Serpanos YC, Wheaton MA. Hearing screening in a well-infant nursery: profile of automated ABR-fail/OAE-pass. Pediatrics. 2011;127(2):269275. 